\title{
Multi-scale visual analysis of trauma injury
}

\author{
Celina Imielinska ${ }^{1,2}$ \\ Andrzej Przekwas ${ }^{3}$ \\ XG $\operatorname{Tan}^{3}$
}

${ }^{1}$ Department of Biomedical Informatics, New York, NY, U.S.A.; ${ }^{2}$ Department of Computer Science, Columbia University, New York, NY, U.S.A.; ${ }^{3}$ CFD Research Corp., Huntsville, AL, U.S.A.

\section{Correspondence:}

Celina Imielinska,

Department of Biomedical Informatics and Department of Computer Science, Columbia University, 701 W. 168th Str., HHSC 201, New York,

NY10032, U.S.A.

Tel: 2123051440 ;

Fax: 212305 8388;

E-mail: ci42@columbia.edu

\begin{abstract}
We develop a multi-scale high-fidelity biomechanical and physiologically based modeling tools for trauma (ballistic/impact and blast) injury to brain, lung and spinal cord for resuscitation, treatment planning and design of personnel protection. Several approaches have been used to study blast and ballistic/ impact injuries. Dummy containing pressure sensors and synthetic phantoms of human organs have been used to study bomb blast and car crashes. Large animals like pigs also have been equipped with pressure sensors exposed to blast waves. But these methods do not provide anatomically and physiologically, full optimization of body protection design and require animal sacrifice. Anatomy and medical image-based high-fidelity computational modeling can be used to analyze injury mechanisms and to optimize the design of body protection. This paper presents novel approach of coupled computational fluid dynamics and computational structures dynamics to simulate fluid (air, cerebrospinal fluid)-solid (cranium, brain tissue) interaction during ballistic/blast impact. We propose a trauma injury simulation pipeline concept staring from anatomy and medical image-based high-fidelity 3D geometric modeling, extraction of tissue morphology, generation of computational grids, multiscale biomechanical and physiological simulations, and data visualization. Information Visualization (2006) 5, 279-289.

doi: | 0.1057/palgrave.ivs.9500। 37
\end{abstract}

Keywords: Multi-scale data visualization; medical simulation; physiologically based modeling; medical image segmentation

\section{Introduction}

Primary blast injury (PBI) results from an interaction of pressure wave with the human body, and gas-filled organs, ear, lungs and gastrointestinal track. A typical explosion creates a shock wave traveling at three to five times the speed of sound. Temperature across the shock wave can increase over $1000^{\circ} \mathrm{C}$ near the explosion and pressure can increase abruptly to more than $20 \mathrm{~atm}$ (Figure 1). Closed spaces such as rooms and street canyons cause reflections and shock wave interference patterns that can amplify pressure changes. A large percentage of fatal blast injuries result from PBIs, in which the shock wave directly damages the lungs through violent, localized pressure changes. Because a shock wave travels faster in liquids and solids than air, organs like the lungs, intestines and inner ear are damaged by shear stresses when a shock wave reaches tissue-gas interfaces. The lungs are most vulnerable to PBIs because they contain large surface areas of fragile alveoli where oxygen is exchanged for carbon dioxide. Differences in wave propagation mechanics at the interface between air and blood in alveolar membranes cause large deformations of lung tissue that collapse alveolar sacs, tear alveolar membranes and rupture blood vessels. The extent of lung injury is a decisive parameter for mortality in victims surviving an explosion. Because the symptoms of PBI are often delayed, victims may not receive timely treatment.
Received: 20 January 2006

Revised: 27 July 2006

Accepted: 16 September 2006 

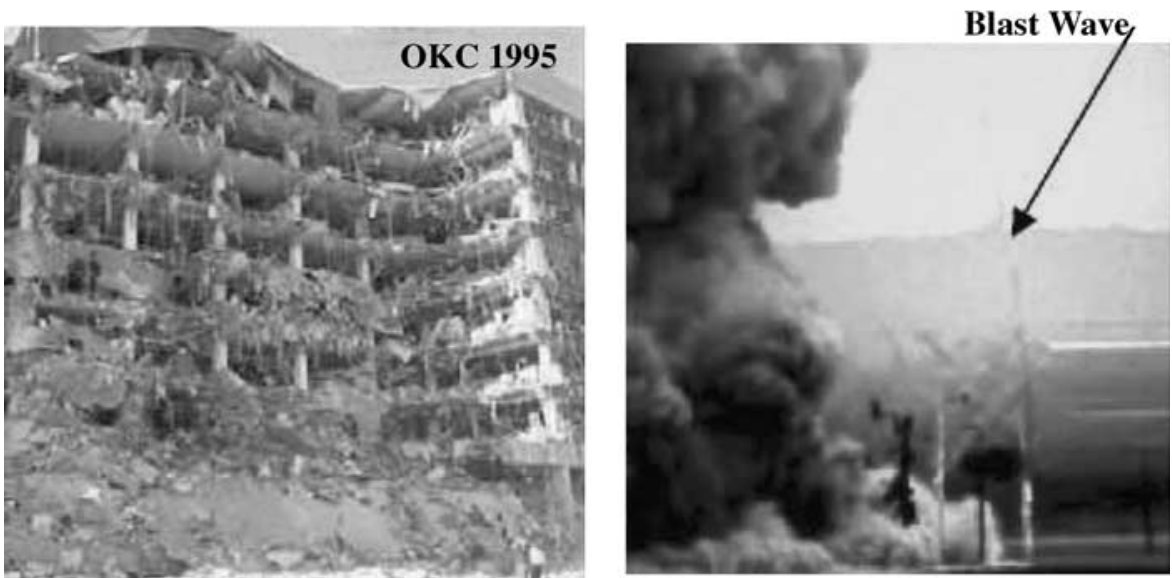

Figure 1 Blast wave dynamics - injury potential.
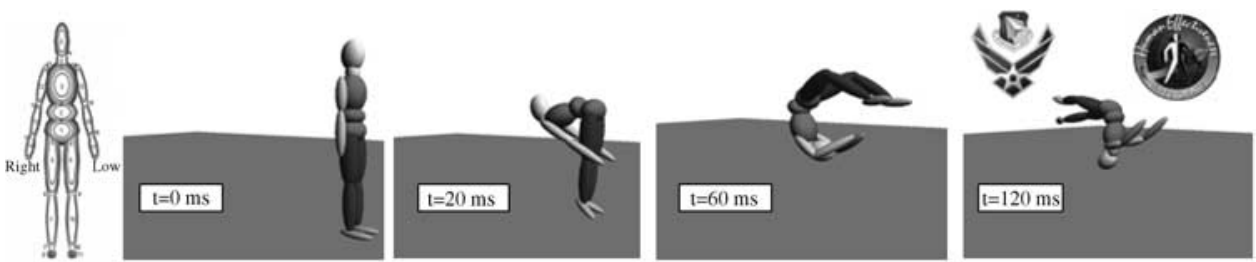

Figure 2 Modeling of body blast dynamics with FEM (CFDRC).

We are seeking to mathematically model blast wave interaction with human body and ballistic/impact injury to the vital human organs, as shown in Figure 2, where body blast dynamics has been modeled with finite element model (FEM) ${ }^{1}$ and using articulated human body model developed at CFDRC. We are in a process of designing a multi-scale fidelity biomechanical and physiologically based modeling tools for trauma (ballistic/impact and blast) injury to brain, lung and spinal cord. This modeling effort requires a combination of diverse disciplines including gas and human body dynamics, tissue biomechanics and the pathophysiology of organ damage.

In this paper we describe our preliminary results from our pilot head trauma project that illustrate the key steps in trauma injury simulation pipeline, starting from anatomy and medical image-based high-fidelity 3D geometric modeling, extraction of tissue morphology, generation of computational grids - volumetric meshes, multiscale biomechanical and physiological simulations, and data visualization.

\section{Brain anatomy and neurophysiology-macro view}

Brain is protected by the skull (bone) surrounded by the scalp that is about $5-7 \mathrm{~mm}$ thick. Between skull and brain there are three membranes: dura, arachnoid and pia matter. The membranes are separated from each other by subdural and subarachnoidal spaces filled with the cerebrospinal fluid (CSF). Blood vessels - bridging veins - cross the
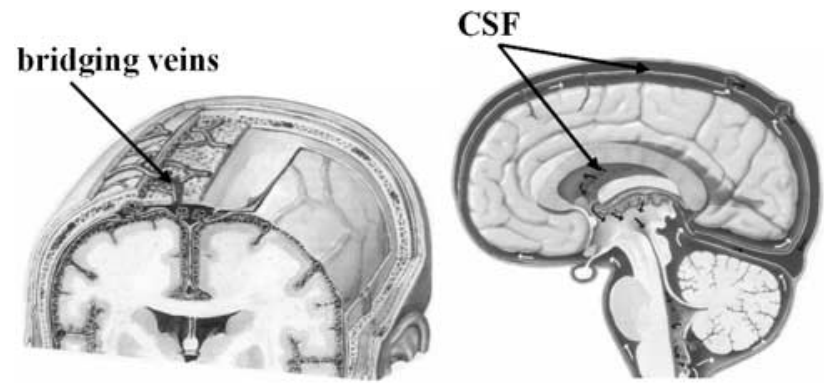

Figure 3 Brain anatomy (public source for images).

membranes. CSF and bridging veins play an important role in the head shock-absorbing capacity. Brain cerebral hemispheres are divided into lobes made up of gray/white matter and they encompass the ventricles where the CSF is formed. Arterial blood enters the brain via two internal carotid and two vertebral arteries (Figure 3).

\section{Pathophysiology of traumatic brain injury (TBI)}

The ballistic impact or a shock wave can cause the helmet shell to impact the human head and impart a transient pressure loading on the head, resulting in blunt trauma. Depending on the force magnitude and duration, there are several biomechanical, hemodynamic and 


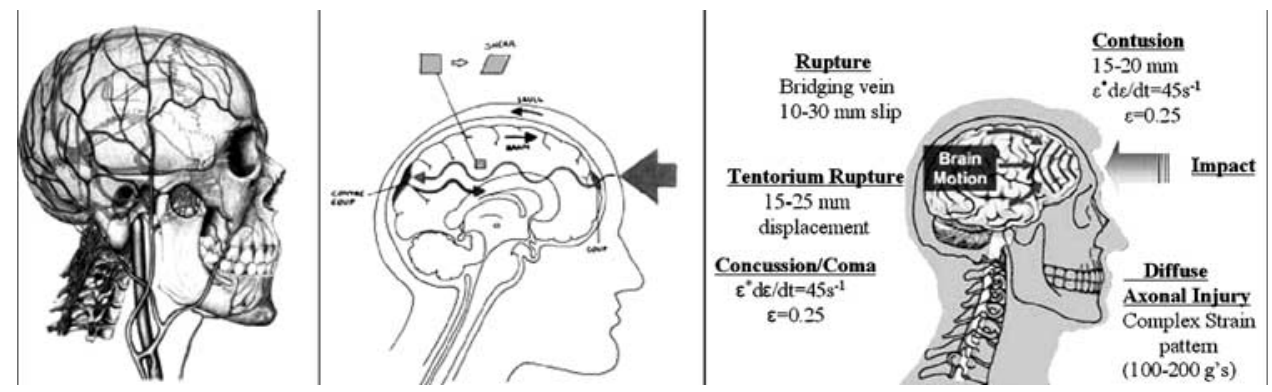

Figure 4 Human skull with veins, ballistic impact TBI mechanism (public source for images).

neurological consequences: (a) pressure wave propagation in brain tissue may case large deformation within and between gray and white matter. It will couple to brain tissue by skull deformation, and may cause local cavitation in sinuses during skull wall recoil; (b) inertial impact of the brain tissue on the skull, brain-skull contact, deformation and breakup of the brain matter tethering points, such as blood vessels, cranial nerves, as well as deformation and overstretching of neural axons; (c) subarachnoid and subdural hemorrhages, edemas, hematomas pushing on the (c) brain tissue: (d) neurological, immune and metabolic responses following the mechanical trauma, and others.

Figure 4 presents the skull with major veins, the biomechanical events of traveling pressure and shear waves in the brain tissue, and types of the brain injury resulting from a high-energy frontal focal impact on a head.

Non-penetrating TBI is commonly categorized as either diffuse or focal. Diffuse injuries are widespread and range from mild concussion to diffuse axonal injury DAI. Diffuse injuries also include hypoxic brain damage, brain swelling and diffuse vascular injury. Focal injuries are more localized and are typically associated with blood vessel failure (hemorrhage) or uncontrolled water transport (edema) resulting in blood pools that in turn may result in severe secondary damage due to increasing intracranial pressure and distortion of the brain mass. Movement of brain within the skull may cause tears in veins that bridge the brain and the skull and can force the brain tissue against bony protuberances inside the cranium. There are also hosts of metabolic and inflammatory responses such as hypoxia, ischemia and production of toxic reactive oxygen species. At the cellular level, TBI causes axonal tears within the white matter resulting in immediate disconnection of axons (primary axotomy) and, more importantly, secondary disconnection over an extended period of time (secondary axotomy).

\section{Material and methods}

Computational modeling of head biomechanics and TBI in particular requires 3D FEM, that is, geometry and computational mesh, of the human head. The model should include not only geometry but also identification of material types, for example, skull bone, brain tissue gray and white matter, CSF, meningeal members, major blood vessels, etc. Such a model can be obtained only from medical images and its creation requires significant effort and expertise. Several groups worldwide have spent large amount of effort in generating 2D and 3D head/brain FEM models. However, no one has established yet a benchmark human head grid of desired accuracy and fidelity to perform large-scale biomechanical simulations. Thus, generation of 3D FEM meshes from medical images is one of the most challenging tasks in highfidelity computational biomechanics. It is not unusual to spend several weeks or months to generate an anatomically correct, good quality mesh, for brain, thorax or spine.

We demonstrate a 3D modeling pipeline starting with acquisition of data from various imaging modalities that can provide the most detailed anatomy to FEM modeling. In this pilot project, we demonstrate a prototype software tool, image-based meshing pipeline, on automated generation of human head anatomy, triangulated surfaces, and 3D tetrahedral meshes from segmented medical imaging data. We showed automated mesh coarsening from original millions of triangles to an arbitrary small number of triangles (as small as 30,000 and even 5000). This technology is critical for cost- and time-effective FEM computational biomechanics.

High-fidelity simulation combining right mathematical models describing body dynamics, tissue biomechanics and injury pathophysiology requires detailed geometry and material properties of the organs (torso/lung, head/brain). Such information has to be extracted from anatomical data and multimodal medical imaging. Computational models have to be calibrated and validated against available experimental models such as physical surrogates and animal 'models'. The computational model will be invaluable in better understanding of injury mechanisms, in better planning of experimental study and in optimized design of personnel protection armor.

\section{Multiscale modeling approach - geometry and meshing} Based on medical image data, we generate 3D geometry of human organs: for example, a 3D model of human 


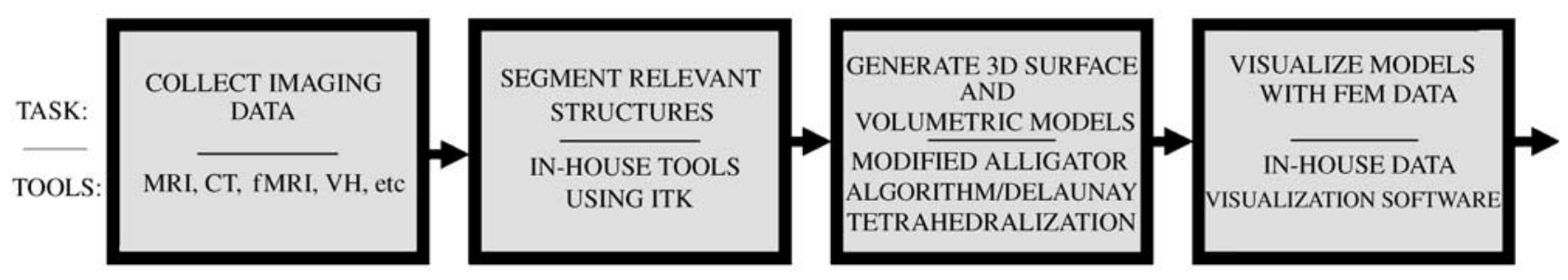

Figure 5 Key Tasks and tools in image processing and model generation pipeline.

head, including skull, brain tissues, ventricles, major arteries and veins. We define material volume conditions for bone, CSF, white and gray matter brain tissue, and generate 3D FEM computational mesh. Truss model is used to represent neck muscles, and $\mathrm{CoBi}$ 'embedded wires' to model vascular perfusion.

\section{Design of imaging system for organ injury}

We define a 3D imaging pipeline that provides image acquisition, processing, segmentation and modeling of 3D anatomy of human anatomical structures. The images come from CT, MR patient scans and also from CT and color cryosections of the Visible Human data sets. Methods for function and physiology are applied to depict, assess and classify the full extent of brain, spine and lung injury under trauma. The structures are later used for FEM impact and biomechanics simulations.

Image-based head geometry/mesh generation pipeline We are developing a general purpose image processing and mesh generation pipeline that will take as inputs medical imaging data of the head from a variety of different imaging modalities, such as the Visible Human data set, MRI, CT, fMRI, etc., and segment the data to produce surface and volumetric models of the relevant anatomical structures. It is important to note here that the surface and volumetric models need to be of sufficiently high quality, that is, suitable for engineering simulations (and not just visualization). This means that the generated surface meshes are properly connected, closed where needed, have no overlapping or dangling triangles, and so on. Another complication is that the surface meshes tend to be very dense, and that if those surface meshes are directly used in the volumetric mesh generation process, one may end up with millions of cells in the volume mesh. Such a large mesh makes the subsequent FEM analysis process intractable. A computational mesh of $20 \mathrm{~K}$ to $100 \mathrm{~K}$ cells is considered optimal for FEM analysis. The pipeline also includes interactive visualization software that can be used to examine combinations of different 3D anatomical models and will link to finite element solvers, like CoBi, in order to create and present the results of FEM simulations. The pipeline will serve as the foundation for a variety of medical simulations. The full visualization suite makes use of unique image segmentation, model generation and interface technologies developed in-house. The system will provide high-resolution 3D anatomical models for finite element simulations and will give users an intuitive tool for examining all aspects of the skull-brain/helmet-head interaction during trauma.

As shown in Figure 5, the overall image processing and model generation pipeline consists of the following stages. First, medical imaging data is acquired from human subjects and cadavers. The data can come from a wide array of imaging devices, including MRI, fMRI, CT, X-ray and others. Next, the system processes the data using image segmentation modules implemented using the ITK segmentation toolkit, which shows the main steps in the pipeline being applied to structures in the human cervical spine.

Hybrid segmentation Over the past several years a variety of segmentation methods have been developed. Boundary-based techniques such as snakes ${ }^{2}$ start with a deformable boundary and attempt to align this boundary with the edges in the image. The advantage here is that image information inside the object is considered as well as that on the boundaries. However, there is no provision in the region-based framework for including the shape of the region, which can lead to noisy boundaries and holes in the interior of the object.

Like several other recent approaches, ${ }^{3,4}$ our design integrates the boundary and region-based techniques into a hybrid framework. By combining these, we gain greater robustness than found in either technique alone. Most of the earlier approaches use prior models for their regionbased statistics, something we would rather avoid in order to increase usefulness in situations where a comprehensive set of priors may not be available.

We have recently developed, under the IT segmentation toolkit, ${ }^{5}$ a new segmentation method that integrates the deformable model ${ }^{4}$ with fuzzy connectedness, 6 and the Voronoi Diagram (VD) classification. ${ }^{7}$ We have tested this method on the Visible Human and clinical data. This hybrid method ${ }^{5}$ requires minimal manual initialization. We start with the fuzzy connectedness algorithm to generate a region with a sample of tissue. From the sample, a homogeneity operator is automatically generated. The homogeneity operator is used by the VD classification to 

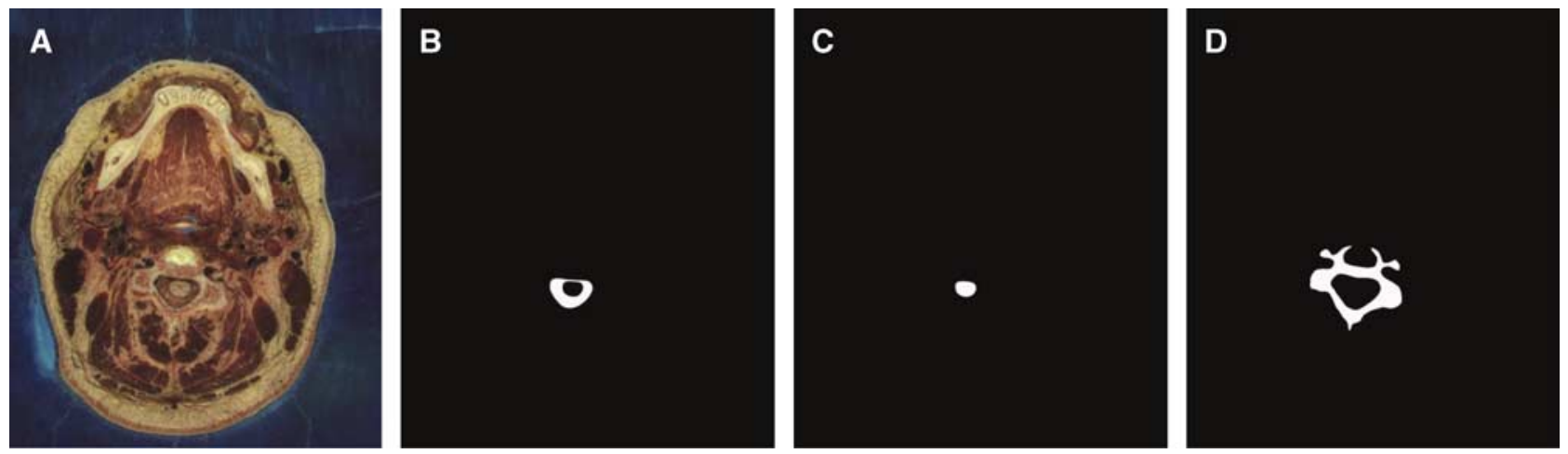

Figure 6 Segmentation of C3 vertebral body in cervical spine, CSF and spinal cord: (A) color cryosection Visible Human data; (B)-(D) segmentation of CSF, spinal cord and C3 vertebra, respectively.

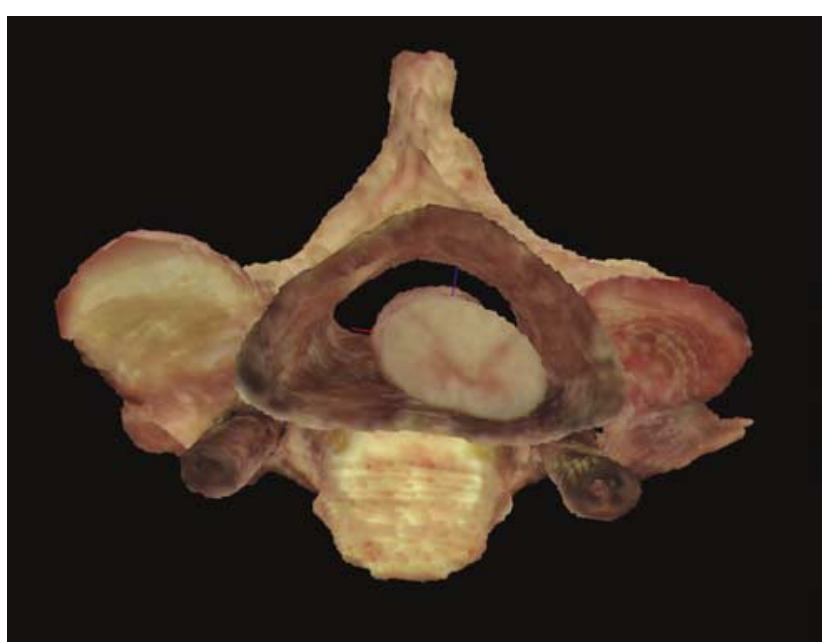

Figure 7 3D models of a vertebra with dura and spinal cord from the Visible Human data visualized with 3D Vesalius Visualizer. ${ }^{[8]}$

produce an estimation of the boundary. We apply the deformable model to determine the final boundary.

Modeling head anatomy We use two sets of data depicting head anatomy: (a) the Visible Human Male/Female color cryosection data segmented (Figure 6) and reconstructed/rendered with Columbia University in-house 3D Vesalius Visualizer, 8 (Figure 7). (b) Visible Human Male CT (Figure 8) segmented with hybrid segmentation method ${ }^{5}$ (Figure 9) and reconstructed with t-shells ${ }^{9}$ (Figure 10).

The segmented data serves as the inputs to a mesh generation component that uses a variant of the reconstruction technique known as the alligator algorithm to create high-resolution surface meshes.

\section{Geometry preserving automated coarsening of triangulated} surfaces High-resolution medical images and image processing software generate large number of surface triangles. This is desired for the image visualization but is not practical for FEM biomechanics modeling. It is not uncommon to see millions of triangles on the cranium and brain surfaces. A FEM tetrahedral volume mesh generated from such number of surface mesh would have hundreds of millions elements. We have developed a novel algorithm and a software module for automated coarsening of triangulated surfaces while preserving the geometrical fidelity. We have tested it on automated coarsening of the surface triangle meshes for a human head depicted in Figure 11. Figure 11A-C present the original human head mesh with 950,000 triangles and two levels of coarsened grids. The algorithm provides very powerful capability of generating coarse tessellation, which can be used directly for tetrahedral meshing or as a surface geometry for generation of NURB surfaces and further high-quality structured FEM grids. In the future, we plan to implement this module into a data processing software framework for semi-automated generation of high-quality FEM grids for human body, with emphasis on human head, neck and spine.

The next step in the pipeline is the generation of the volume mesh. The input is the coarsened surface mesh from the previous step. We are using a tetrahedral mesh generator that combines the Advancing Front and Delaunay mesh generation methods. The mesh generator allows the specification of surface and volume conditions to be directly specified on the geometry. From there the 3D model can be output into a desired model format. Still a reduced surface mesh, from which a volumetric mesh is derived, may have artifacts that have to be repaired before it will be turned into a volumetric mesh. In Figure 12, we show a process of coarsening, repaired mesh through a process of 'shrink wrapping' with mathematically correct mesh of specified (lower) density, since an optimal surface mesh can be easily reduced, and a corresponding tetrahedral volumetric mesh. A volumetric mesh can be generated for a single slice using CFDRC Micromesh Tool, as shown in Figure 13.

Finally, the resulting models can be viewed and manipulated in an interactive $3 \mathrm{D}$ visualization program that can also pass data to and from finite element simulations 

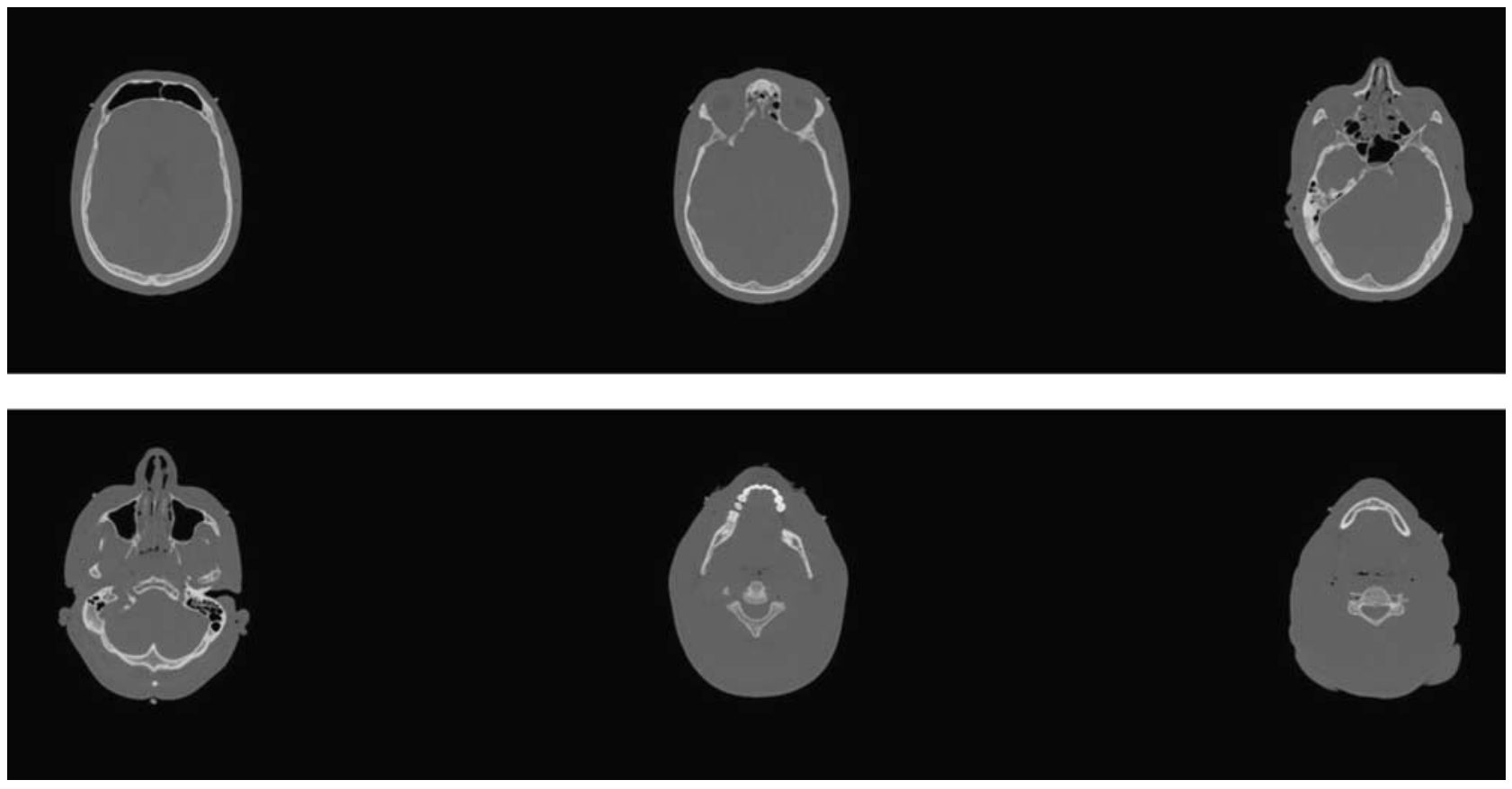

Figure 8 Visible Human Male CT input data.
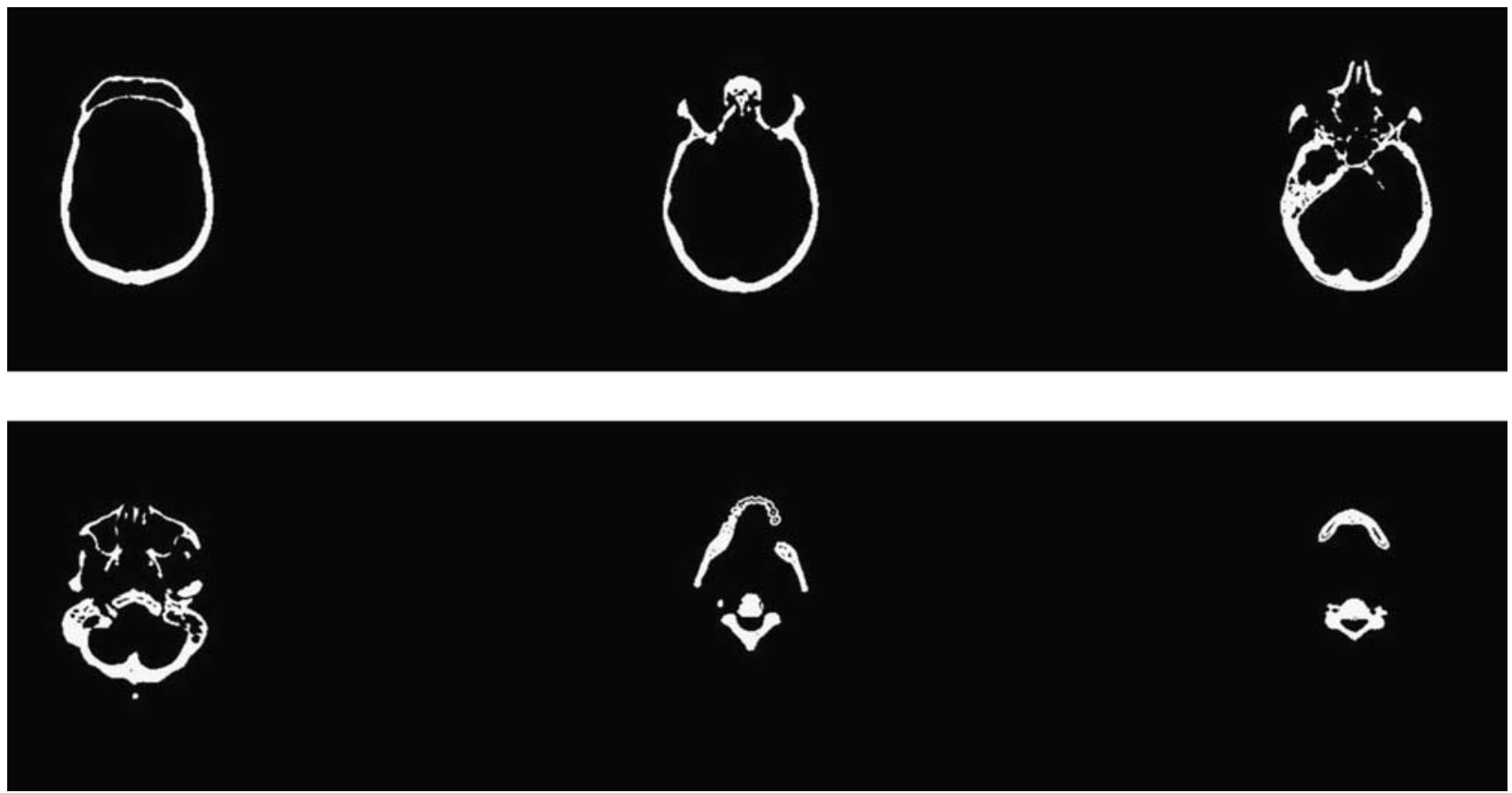

Figure 9 Visible Human Male CT segmented data.

in order to modify simulation parameters and visualize the results. The pipeline described above is highly flexible and extensible, so new algorithms and visualization options can easily be added to the basic framework. All of the requisite components already exist but at various stages of maturity. The image acquisition, segmentation and surface mesh generation modules are robust and stable. 


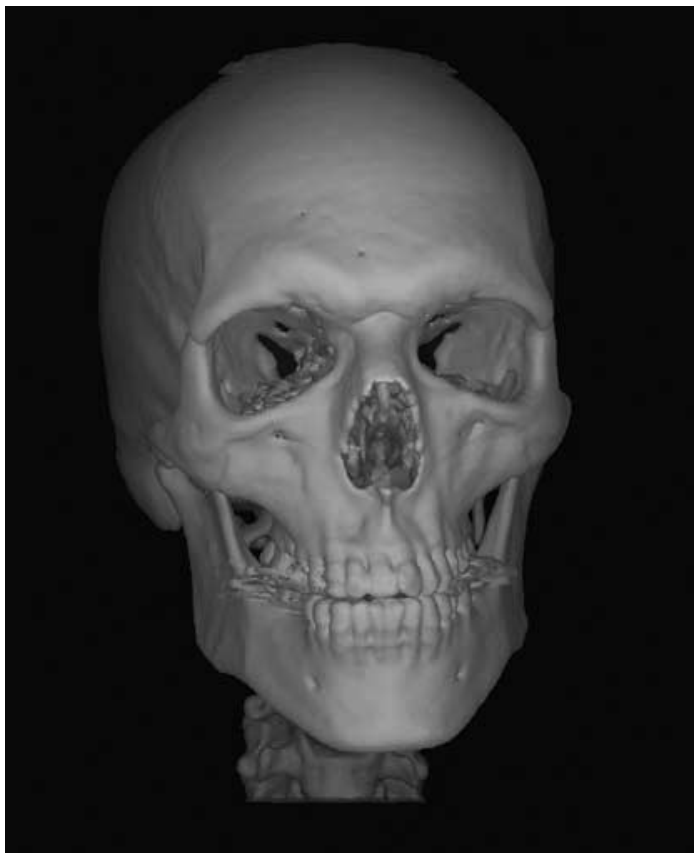

Figure 10 Visible Human Male skull reconstructed with t-shells. ${ }^{9}$

\section{Adaptation of FEM model for ballistic injury}

CFDRC is developing software tools FEM-Bi for multi-scale modeling of blast injuries to thorax, lungs, brain and spine coupled to physiological trauma injury model CoBi. We are adapting FEM-Bi for modeling ballistic impact of head protective gear materials in proximity to animal/human head and for modeling head injury. The FEM model can be validated on existing experimental data of mechanical behavior of material used in the protective gear and protection padding.

\section{Explicit dynamics systems}

In the current helmeted head model, larger number of finite elements (100K or more) is needed to properly resolve the complex geometry and stress/strain localization. As a result, the solution time for the implicit FEM solution for large grid counts $(>100 \mathrm{~K})$ will increase dramatically and become prohibitively expensive. To speed up the computations and take less computer memory, we developed an explicit solver for the FEM equations. For large-scale highspeed event, it is advantageous to use the conditionalstable explicit solver because: (1) A small time step is required to track the high-frequency portion of the response anyway, making it impossible to take advantage of the large time steps permitted by implicit methods. (2) Time integration of the discrete momentum equations does not require the solution of any equations due to the use of diagonal mass matrix. The construction and solving of linear system in the implicit method in each iteration of time step is very time-consuming, especially for the large FEM. (3) Robustness for the non-linear problem compared to the Newton iterations in non-linear implicit solver, in which the convergence failure can often occur due to 'rough' events such as contact-impact or the large time step.
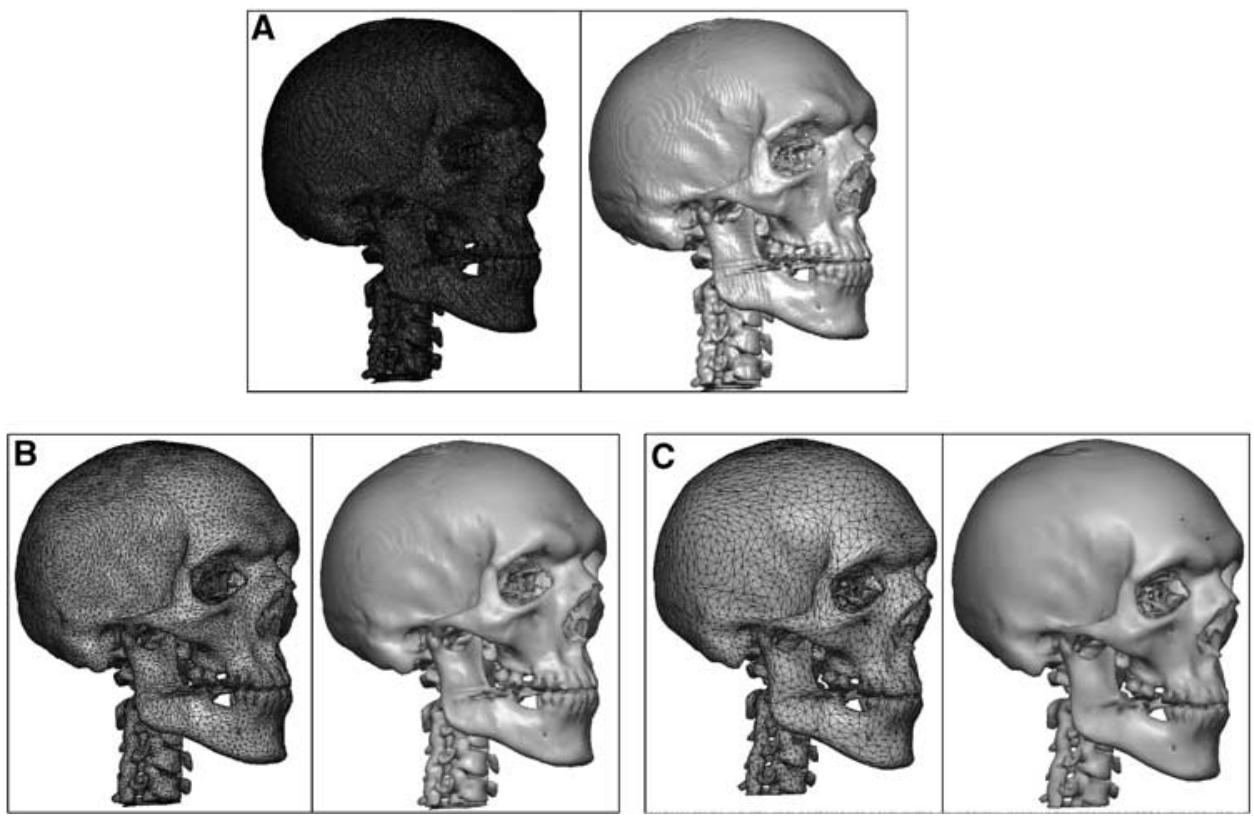

Figure 11 (A) Original model with 950,000 triangles (too fine to see) and rendered solid model, (B) coarsened mesh with 95,000 triangles and rendered resultant solid model and (C) coarsened mesh with 37,500 triangles and rendered resultant model. 

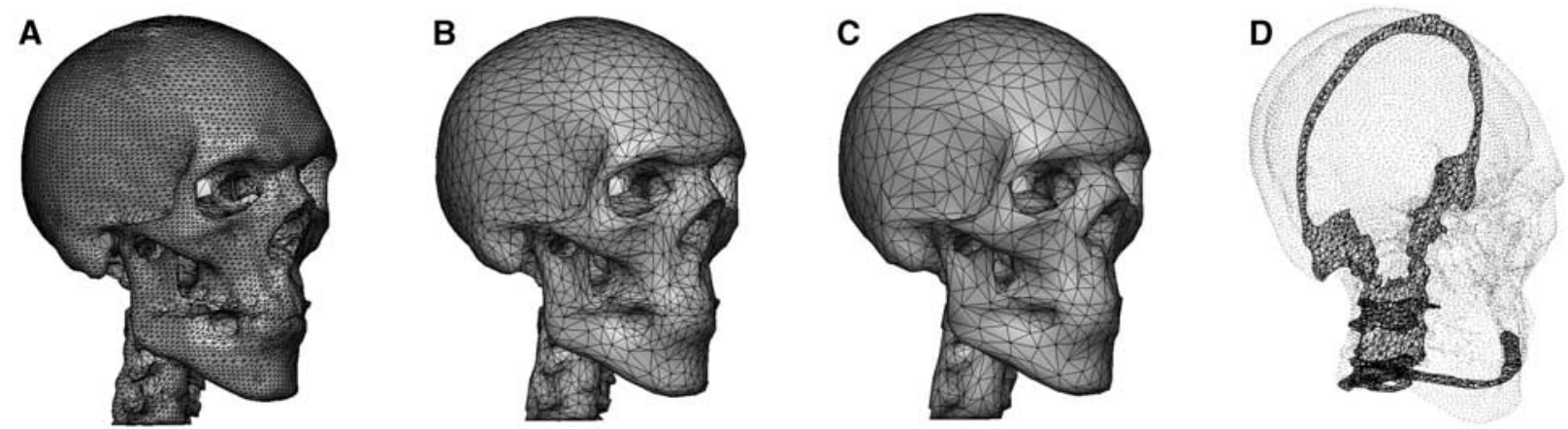

Figure 12 Repaired, optimal mesh, reduced using shrink wrapping process: (A) 50,0000 triangles, (B) 10,000 triangles, (C) 5000 triangles and (D) tetrahedral volumetric mesh with 150,000 element.
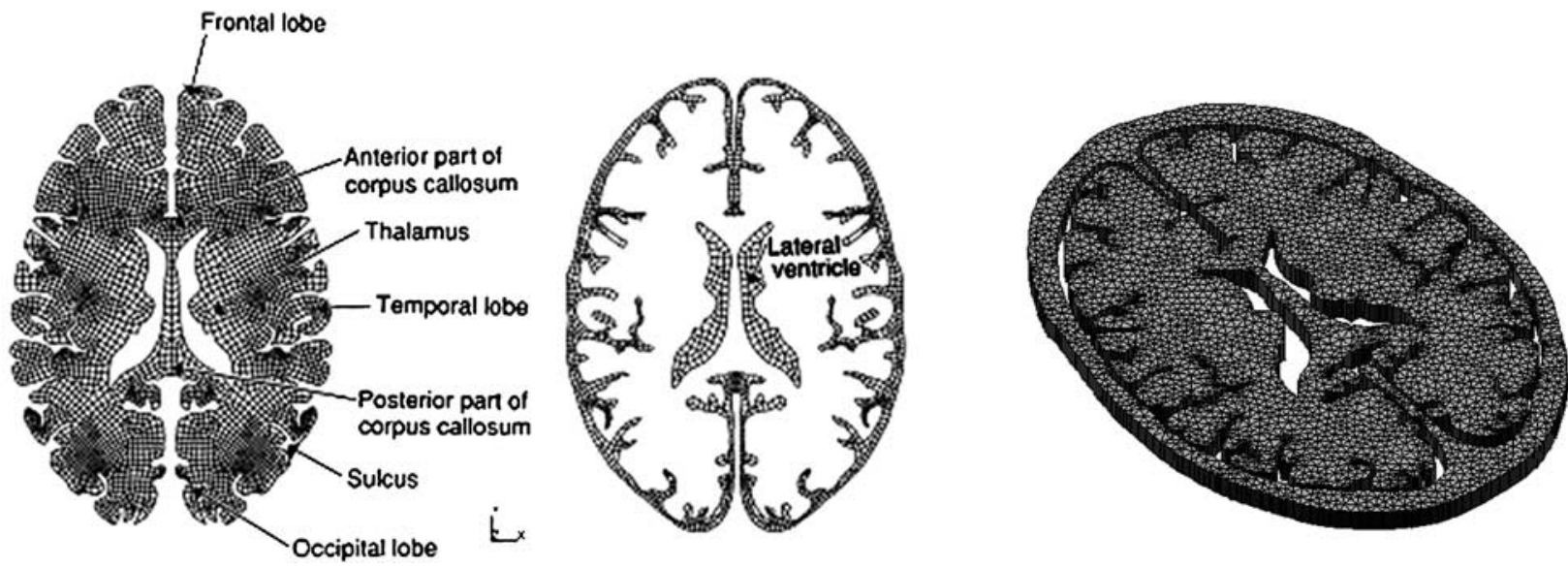

Figure 13 Volumetric mesh of axial brain section generated with CFRC Micromesh Tool.

\section{Explicit solution of frictional contact problem}

The development includes the virtual work and contact searching, which appears to be highly desirable for the general robustness of explicit finite element techniques. Since the explicit formulation has no convergence problem caused by the non-linear iterations, the resulting frictional contact model is suitable for applications displaying significant non-linear behavior. An important aspect of contact problems is the method used to convert the associated variational inequality to an equality suitable for finite element solution. In the explicit analysis, we still adopt the penalty method since it offers distinct advantages on the integration algorithm for the frictional tractions, compared to the Lagrange multipliers method. At beginning of each time step, the estimate for an appropriate choice of the penalty parameter is based on the material properties and geometric size of the shell element. The increasing of the default penalty parameter becomes necessary if the results show visible penetration, and thus does not fulfill the constraint equation in a correct way. On the other hand, the larger penalty parameter may decrease the explicit time step size.
The most important practical aspect of computing the contact force vector is acquisition of the projection for the finite element node, that is, the contact searching. Briefly stated, the algorithm consists of the following three phases: (1) identification of the closest target node for the finite element node; (2) among all target elements sharing the closest node, identify the element containing the projection and (3) calculate the coordinates of the projected point. In the current implementation, we calculate the closest target node once at the beginning of each global time step, which is used for the fluid domain in the fluid-structure interaction (FSI) simulation. During one global time step, the local search for the projection is conducted for each finite element explicit time step.

\section{Parametric simulations}

We will use in the future the medical imaging data and resulting 3D models to set up parametric model of the head, spine and other anatomical regions. We will perform ballistic impact FEM simulations and analyze the dynamics of protective gear in contact with underlying anatomy, 


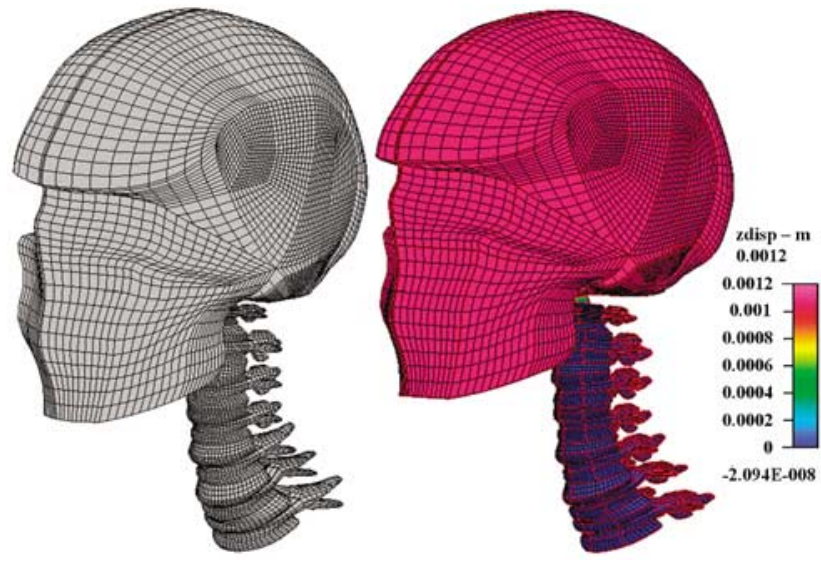

Figure 14 The head-spine FEM and explicit solution for the head-spine model at $t=0.12 \mathrm{~ms}$.

Table 1 CPU time and memory requirements of explicit finite element solvers for one time step

\begin{tabular}{lcc}
\hline Model size (no. of d.o.f.'s) & CPU (s) & Memory (MB) \\
\hline 155,394 & 16.1 & 150 \\
\hline
\end{tabular}

the material-structure impact loads and potential injury to the anatomical structure(s).

\section{Results}

In the following, we show examples to verify the correctness of the new developed explicit finite element solver, and the newly developed truss model used in the gear protected head under impact.

\section{Test on explicit finite element solver}

We have implemented the explicit solver of the FEM for the FSI applications, in addition to the existing implicit solver. Here are some preliminary results to show the effectiveness of the developed explicit solver. The key features of the current explicit solver implementation include:
(1) optimal explicit time step size to maintain numerical stability and accuracy, (2) global/local search algorithm for the contact problem, (3) full-integrated element to avoid the hourglassing modes and (4) numerical dissipation for the central difference time stepping.

The new explicit formulation for the structures calculations was then tried out on a large model with 44,791 eight-node solid element (155,394d.o.f.'s) as shown in Figure 14 . We made the finite element mesh from the geometry data provided by Horgan and Gilchrist. ${ }^{10}$ The finite element takes into account the cerebrum, cerebellum, falx and tentorium, CSF, dura, three-layered cortical and trabecular skull bone, scalp, and facial bone. Table 1 shows the CPU time and computer memory needed for the test case, in which the head is moving forward with an initial velocity. The stable time step size for the explicit algorithm is calculated internally and adjusted.

\section{D Head-spine model under blunt impact}

To test the computational method a 2D multi-body test problem comprised of a helmet-head-cervical spine under rear impact has been setup. A rigid projectile is impacting the outer surface of the helmet with initial velocity of $30 \mathrm{~m} / \mathrm{s}$. The helmet, protection pad, head and C0-T1 spine are approximately modeled with $2 \mathrm{D}$ solid elements. The neck muscle and the ligament between vertebrae are modeled with the viscoelastic truss element. The bottom surface of T1 vertebrate is fixed. In Figure 15, as the projectile is decelerated and rebounded during the impact on the helmet, both the helmet and the protection pad are deformed and rotated along the skull, and part of the absorbed energy by the helmet is transferred to the entire head in the form of rapid, typically angular acceleration of the head. Severe injuries of the skull, brain and cervical spine can be identified under this impact based on certain head injury criteria such as the critical strain/strain rate at the tissue level.

It is noted that since we model the CSF between the skull and the brain as the solid elements, the motion of the brain may be more constrained than in the real situation. We will report in the near future how the brain deforms surrounded by CSF modeled with the fluid elements.
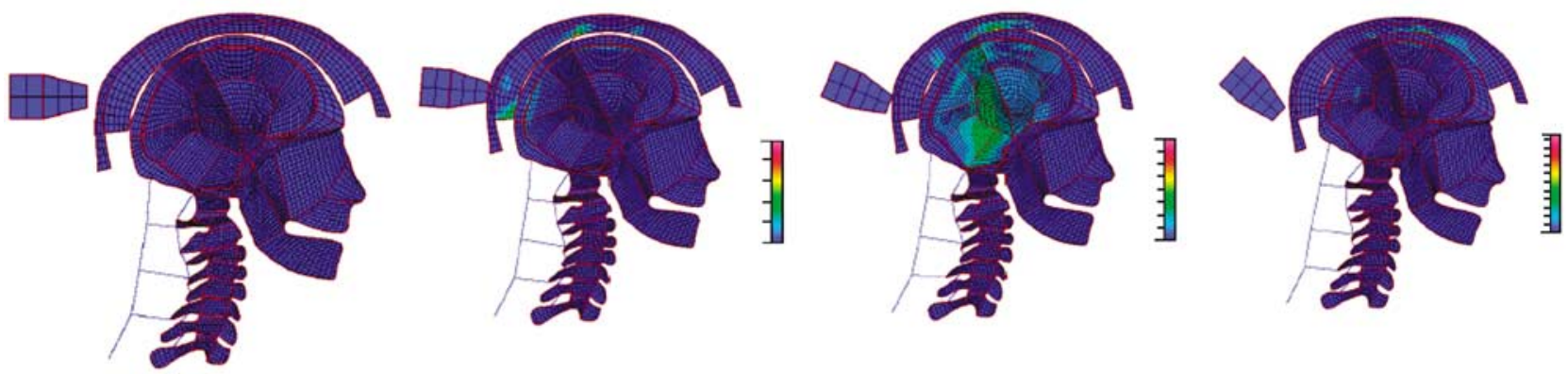

Figure 15 Solid-truss multi-body system under impact at time (1) $0 \mathrm{~ms}$, (2) $0.5 \mathrm{~ms}$, (3) $1.2 \mathrm{~ms}$ and (4) $1.8 \mathrm{~ms}$. 

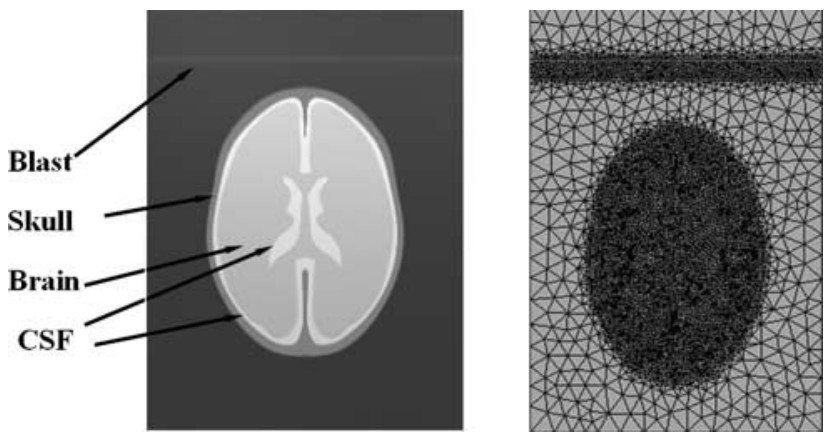

Figure 16 Segmentation of a 2D cross-section of human head and a computational mesh for blast wave dynamics simulations.

\section{Coupled fluid--structure simulation in head injury events}

In reality, head injury due to blast is a complex FSI process (Figure 16). Using the present FSI model, we are able to model the direct impact of the pressure wave on the skull. The skull motion then induces a pressure wave inside CSF, which in turn transfers to the brain causing brain damage. The initial condition is taken as a local high-pressure and high-temperature explosion zone next to the head.

Figure 17 shows the sequence of the blast wave passing through the skull and brain of a human head. Here the pressure wave propagates from the top (or the front of the body) to the bottom. Once the blast wave front reaches the skull, it is subject to a high resistance, which in turn leads to partial reflection of the blast wave from the skull. The other part of the wave compresses the skull wall (moves the wall downward), sending a pressure wave into the CSF. This pressure wave will propagate in all three dimensions inside the skull. The wave in the direction normal to the skull thickness squeezes the CSF fluid inside, causing the local increase of the pressure inside the CSF fluid. This high pressure in turn pushes the brain downward. The sequence clearly shows the transport of wave from blast into the brain stress. The decompression on CSF leads to a local lower pressure zone, which sucks the brain into it and may cause potential damage in this region due to high stress. This pressure wave in the CSF is much faster than that of the pressure wave in the air outside of the skull, since the speed of sound in the skull, brain and CSF is about $4-8$ times faster than that in the air. By $t=1.2 \mathrm{~ms}$, the original blast wave has transported from the air to skull, from skull to CSF, from CSF to brain and from brain to the ventricles.

\section{Software integration}

We will integrate CFDRC software toolkit CoBi for highfidelity multi-scale modeling of head biomechanics and

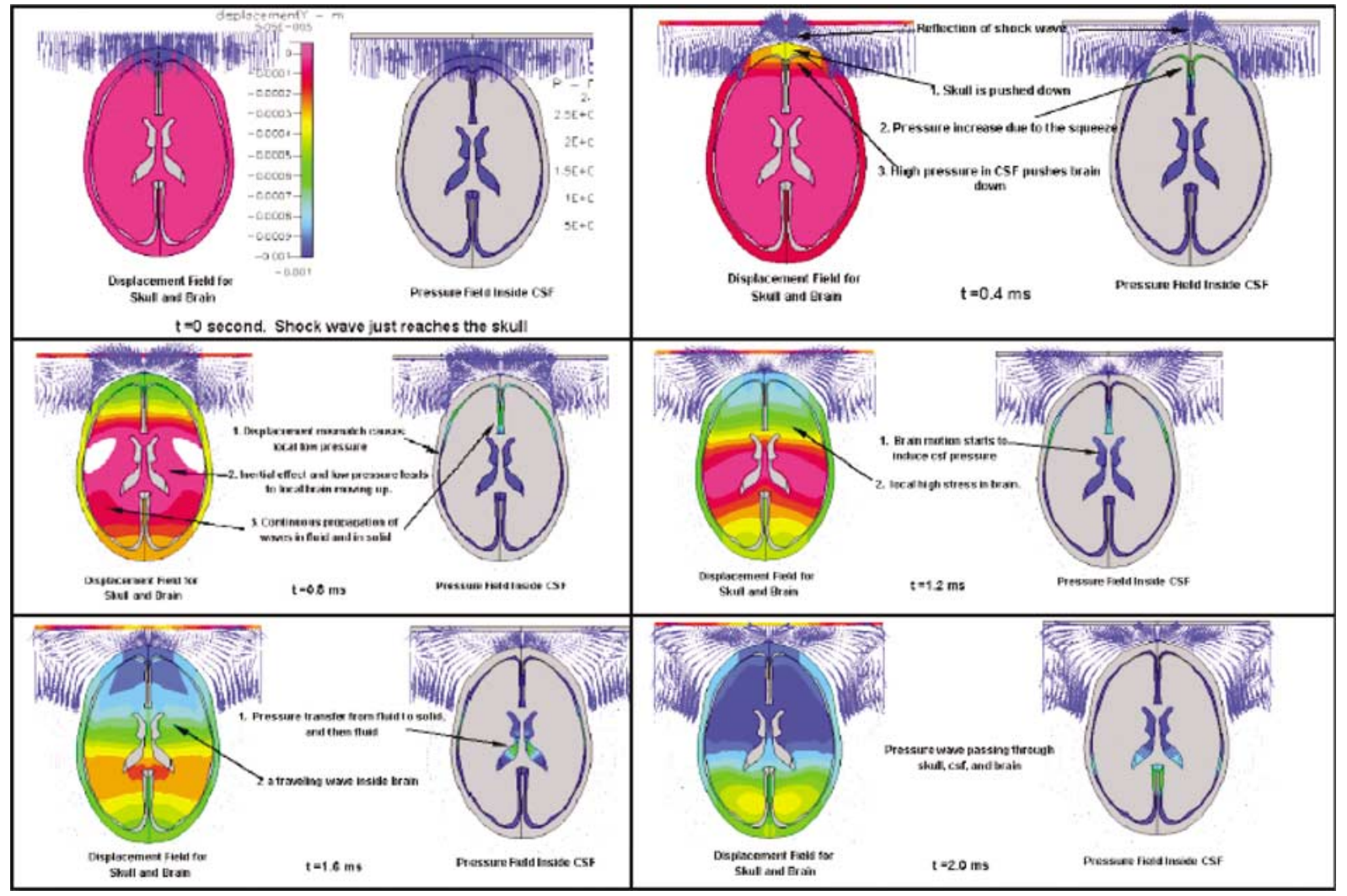

Figure 17 Blast dynamics impact on the brain simulation: displacement, flow and pressure field around the skull and brain. 


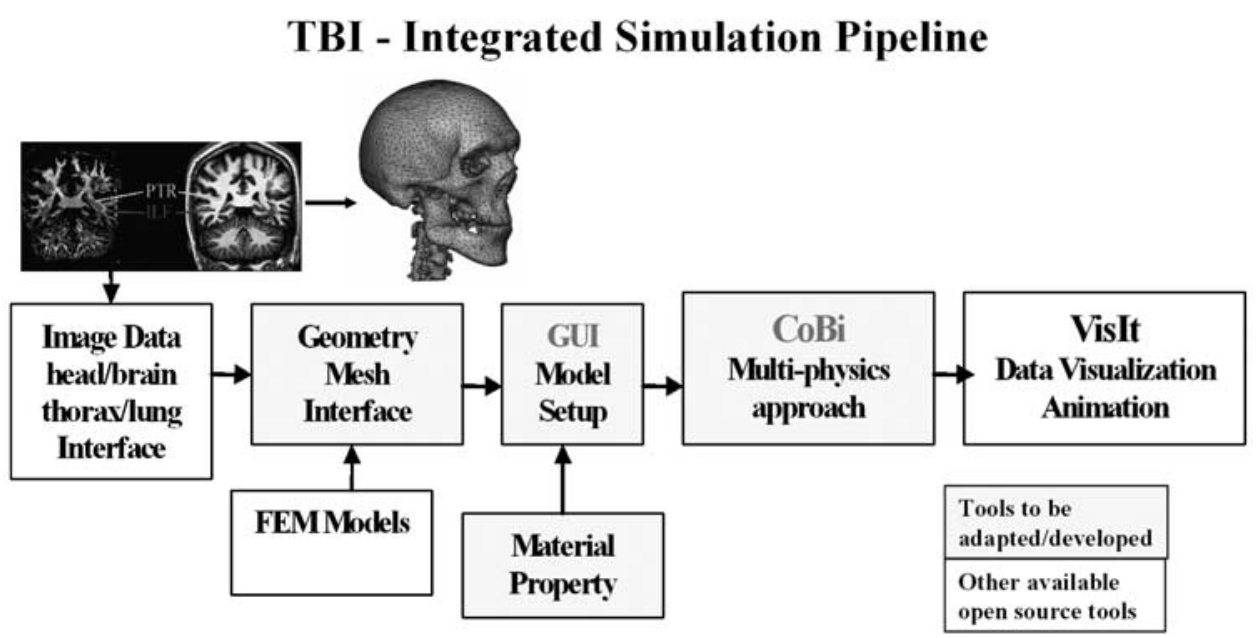

Figure 18 Integrated head biomechanics modeling tool for brain injury simulation.

brain injury for various injury scenarios and mechanisms, as shown in Figure 18. The software environment will include interfaces to medical imaging databases, extraction of geometry/mesh, GUI for parametric model setup, problem execution and data extraction.

\section{Discussion}

We demonstrated a simulation pipeline for modeling trauma to head and spine. We plan to develop robust, generic advanced modeling tools and experimental procedures for modeling trauma under ballistic and explosion blast injury. We would like to develop streamlined procedure to generate good quality FEM (volumetric) meshes for human organs from medical images. Databases are needed with examples of parametric head/neck geometries, and tissue-specific material properties. The overall goal is to develop of tightly coupled FEM-CFD software tool for modeling a wide range of trauma cases.

\section{Acknowledgments}

This research has been partially supported by a grant from U.S. Army Aeromedical Research Lab: USAMRC BAA_05-1 grant and internal grant from Department of Neurological Surgery at Columbia University. We would like to thank Dr. J. Udupa from University of Pennsylvania for letting us use 3DVIEWNIX to model head anatomy from the CT data and Autodesk for generous donation of Maya Software.

\section{References}

1 Bathe K-J. Finite Element Procedures. Prentice-Hall: Englewood Cliffs, NJ, 1996.

2 Kass M, Witkin A, Terzopoulos D. Snakes: active contour models. International Journal of Computer Vision 1998; 1: 321-331.

3 Chakraborty A, Duncan JS. Integration of boundary finding and region-based segmentation using game theory. In: Bizais $\mathrm{Y}$ et al. (Ed). Information Processing in Medical Imaging. Kluwer Academic Publishers: Dordrecht. 1995; 189-201.

4 Jones T, Metaxas D. Image Segmentation based on the Integration of pixel affinity and deformable models. In: Yoo T. (Ed.) Proceedings of the IEEE CVPR (Santa Barbara, CA) IEEE Computer Society, June 1998; 330-337.

5 Imielinska C, Udupa JK, Metaxas D, Jin Y, Angelini E, Chen T, Zhuge Y. Hybrid segmentation methods, In: Yoo T. (Ed.), Insight into Images: Principles and Practice for Segmentation, Registration, and Image Analysis, Peters AK, March 2004.

6 Udupa JK, Samarasekera S. Fuzzy connectedness and object definition: theory, algorithms, and applications in image segmentation. Graphical Models and Image Processing 1996; 58: 246-261.

7 Imielinska C, Downes M, Yuan W. Semi-automated color segmentation of anatomical tissue. Journal of CMIG 2000; 24: 173-180.

8 Imielinska C, Molholt P. Incorporating 3D virtual anatomy into the medical curriculum. CACM 2005; 48:49-54.

9 Grevera GJ, Udupa JK, Odhner D. An order of magnitude faster isosurface rendering in software on a PC than using dedicated, general purpose rendering hardware. IEEE Transactions on Visualization and Computer Graphics 2000; 6: 335-345.

10 Horgan TJ, Gilchrist MD. The creation of three-dimensional finite element models for simulating head impact biomechanics. International Journal of Crashworthiness 2003; 8: 353-366. 
Reproduced with permission of the copyright owner. Further reproduction prohibited without permission. 